\title{
Amplification of optical delay by use of matched linearly chirped fiber Bragg gratings
}

\author{
Changhuei Yang,* Siavash Yazdanfar, and Joseph Izatt \\ Department of Biomedical Engineering, 136 Hudson Hall, Duke University, Durham, North Carolina 27708
}

Received September 2, 2003

\begin{abstract}
We describe the use of a matched linearly chirped fiber Bragg grating (FBG) pair as a key element in an adjustable optical delay line. This delay line has the unique property that the achievable optical group delay is orders of magnitude greater (factor of $10^{2}$ in our experiment) than the actual physical displacement. We demonstrate operation of such an optical delay line over a delay range of $3.5 \mathrm{~mm}$ using a pair of matched 1300-nm chirped FBGs with a bandwidth of $20 \mathrm{~nm}$ each. () 2004 Optical Society of America

OCIS codes: $050.2770,110.4500,120.3180,230.1480$.
\end{abstract}

An all-fiber variable optical delay line capable of achieving millimeter-scale optical group delays at high repetition rates would be useful for numerous applications. Such a system could be used as the reference optical delay line in optical coherence tomography (OCT) systems ${ }^{1-3}$ and (or) other interferometric systems for which low loss, long-term stability, and ease of use are important design considerations. Previous publications have demonstrated optical fiber based variable delay lines based on stretching a long optical fiber coiled around a piezoelectric actuator. ${ }^{4}$ Although this method is practical and simple, it has at least two limitations. First, since glass lacks a crystalline structure, long-term maintenance of an optical fiber under tension deforms the fiber and introduces long-term drift into delay line operation. Second, rapid, repetitive, and large stroke stretching of a coiled optical fiber introduces thermal effects, polarization coherence loss, as well as dynamic birefringence effects.

In this Letter we present an all-fiber variable optical delay line based on a matched pair of linearly chirped fiber Bragg gratings (FBGs). ${ }^{5}$ The pair is arranged so as to compensate for each other's chromatic dispersion. By stretching or compressing one of the FBGs, it is possible to create an optical delay that is orders of magnitude greater than the actual physical stretch. This is because the resonance reflection location for each wavelength on the FBG segment translates down the segment as the FBG is stretched, since the period spacings in the FBG are modified by the stretch. By using short FBG segments $(\sim 1 \mathrm{~cm})$, it is possible for the segments to be bonded directly to a piezoelectric stack. This allows for operation of the FBG under net zero tension conditions. This concept has also been independently conceived, and its theoretical analysis was reported by Lee et al. ${ }^{6}$

A chirped FBG reflects components of a polychromatic wave at different points along its length. Specifically, a location $l$ along the FBG will resonantly reflect the light component of wavelength $\lambda$ where

$$
\lambda=2 n \Lambda(l),
$$

with $\Lambda(l)$ being the local period spacing at distance $l$ along the FBG and $n$ being the effective refractive index of the fiber's core mode. After stretching the
FBG, the local period spacing expands and causes the light component of a specific wavelength to be reflected at a different point along the FBG where the resonance condition is met.

A dispersion-compensated variable optical delay line can be constructed from two matched linearly chirped FBGs and a fiber coupler by sending light into one end of a FBG and using the coupler to direct the reflection into an oppositely oriented identical FBG (see Fig. 1). The second FBG can be subjected to a stress or compression to alter its length by $\delta L$. Mathematically, period spacing $\Lambda_{1,2}$ of the FBGs at location $l_{1,2}$ along the fiber length can be expressed as

$$
\begin{aligned}
& \Lambda\left(l_{1}\right)=\Lambda_{-}+\left(\Lambda_{+}-\Lambda_{-}\right) \frac{l_{1}}{L}, \\
& \Lambda\left(l_{2}\right)=\Lambda_{+}\left(1+\frac{\delta L}{L}\right)+\left(\Lambda_{-}-\Lambda_{+}\right) \frac{l_{2}}{L},
\end{aligned}
$$

where $\Lambda_{+}$and $\Lambda_{-}$are the maximum and minimum fringe periods at the ends of the FBGs without stress, and $L$ is the total FBG length. Equation (2b) describes the fact that a stretch or compression of the

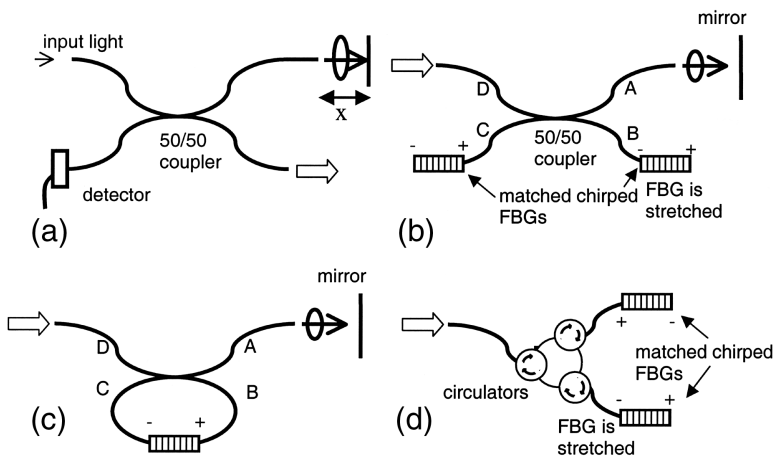

Fig. 1. Interferometer topologies for dispersion-matched amplification of an optical delay by means of stretching FBGs. (a) Michelson interferometer configuration that is common to all topologies. (b) Reference arm configuration for delay amplification by use of a fiber coupler and a pair of FBGs. + and - denote FBG orientation. (c) Reference arm configuration in which a single FBG is employed to demonstrate the potential for near-perfect chromatic dispersion correction given identical oppositely oriented FBGs. (d) Reference arm configuration for delay amplification by use of optical circulators. 
FBG results in a change in period spacing and an increase in the FBG's length leading to an altered reflection condition.

By combining Eqs. (1) and (2), we can derive the optical path delay to be

$$
\begin{aligned}
x_{R}(\lambda, \delta L) & =n_{1} l_{1}(\lambda)+n_{2} l_{2}(\lambda) \\
& =n_{0} L+n_{0}\left(1-p_{e}\right) \frac{\Lambda_{+}}{\Lambda_{+}-\Lambda_{-}} \delta L \\
& \Rightarrow \Delta x_{R}(\delta L)=n_{0}\left(1-p_{e}\right) \frac{\Lambda_{+}}{\Lambda_{+}-\Lambda_{-}} \delta L \\
& \approx n_{0}\left(1-p_{e}\right) \frac{\lambda_{\mathrm{FBG}}}{\Delta \lambda_{\mathrm{FBG}}} \delta L
\end{aligned}
$$

(for $\lambda_{\mathrm{FBG}} \gg \Delta \lambda_{\mathrm{FBG}}$ ),

where $n_{1,2}$ are the respective FBG refractive indices, $n_{0}$ is the refractive index of an unstretched FBG, $\lambda_{\mathrm{FBG}}$ is the FBG center resonantly reflected wavelength, and $\Delta \lambda_{\mathrm{FBG}}$ is the FBG reflection optical bandwidth. Stretching the second FBG also causes a drop in its effective refractive index given by a reduction factor of $\left[1-p_{e}(\delta L / L)\right]$, where $p_{e}$ is the fiber's elasto-optic coefficient (equal to 0.22 for this fiber type). ${ }^{7}$

There are two important notable points in Eq. (3). First, the optical delay is wavelength independent. This implies that, although a single chirped FBG is chromatically dispersive, the dispersion can be significantly corrected by use of two identical but oppositely oriented FBGs. Furthermore, this correction is unchanged even when one of the FBGs is stretched. The second important point to note is that, as $\Delta \lambda_{\mathrm{FBG}}$ is typically much smaller than $\lambda_{\mathrm{FBG}}$, the optical delay change that is due to the stretching of the FBG is much greater than the actual physical stretch by a factor of $n_{0}\left(1-p_{e}\right)\left(\lambda_{\mathrm{FBG}} / \Delta \lambda_{\mathrm{FBG}}\right)$. These two points imply that a dispersion-free variable length optical delay line capable of amplifying a physical stretch into a large optical delay could be constructed.

Figure 1 depicts potential interferometer topologies for dispersion-compensated amplification of an optical delay by means of stretching one of a pair of matched FBGs. To verify that it is possible to create a variable optical delay line as such, we employed the experimental setup shown in Fig. 1(a) with the reference arm configuration of Fig. 1(b). A pair of matched electronbeam etched linearly chirped FBGs obtained from Bragg Photonics (1300-nm center wavelength, >90\% reflectivity over a $20-\mathrm{nm}$ bandwidth, $1.0 \mathrm{~cm}$ long, refractive index 1.47) was used; the second FBG was placed under uniform but adjustable tension by attaching one end of it to a piezoelectric transducer element. A superluminescent diode (OptoSpeed SLED1300D) with a $1300-\mathrm{nm}$ center wavelength, $20-\mathrm{mW}$ power, and FWHM spectral bandwidth of $31 \mathrm{~nm}$ was used as the light source. A scanning mirror was placed in one arm (signal arm) of the interferometer. Light components $(\sim 12.5 \%$ of the light $)$ that travel along paths DBCAD and DACBD are indistinguishable and appropriately dispersion compensated. A more efficient configuration for processing a larger fraction of the light by use of a cluster of optical circulators is shown in Fig. 1(d). The resulting interference signal, $I_{S, R}\left(\Delta x_{S}\right)$, detected for the configuration of Fig. 1(b) is given by

$$
\begin{aligned}
I_{S, R}\left(\Delta x_{S}\right)= & 2 \int_{0}^{\infty} S(\lambda) \sqrt{B(\lambda)} \operatorname{Re}\left\{\exp \left(-i 2 k \Delta x_{S}\right)\right. \\
& \left.\times \exp \left[i 2 k \Delta x_{R}(\delta L)\right]\right\} \mathrm{d} k \\
\approx & 2 \sqrt{I_{R} I_{S}} \cos \left\{2 k_{o}\left[\Delta x_{R}(\delta L)-\Delta x_{S}\right]\right\} \\
& \times \operatorname{sinc}\left\{\frac{2 \pi \Delta \lambda_{\mathrm{FBG}}}{\lambda_{\mathrm{FBG}}{ }^{2}}\left[\Delta x_{R}(\delta L)-\Delta x_{S}\right]\right\} \\
= & 2 \sqrt{I_{R} I_{S}} \cos \left\{2 k _ { o } \left[n_{o}\left(1-p_{e}\right)\right.\right. \\
& \left.\left.\times \frac{\lambda_{\mathrm{FBG}}}{\Delta \lambda_{\mathrm{FBG}}} \delta L-\Delta x_{S}\right]\right\} \operatorname{sinc}\left\{\frac{2 \pi \Delta \lambda_{\mathrm{FBG}}}{\lambda_{\mathrm{FBG}}{ }^{2}}\right. \\
& \left.\times\left[n_{o}\left(1-p_{e}\right) \frac{\lambda_{\mathrm{FBG}}}{\Delta \lambda_{\mathrm{FBG}}} \delta L-\Delta x_{S}\right]\right\},
\end{aligned}
$$

where $S(\lambda)$ is the spectral profile of the light source, $B(\lambda)$ is the reflection spectral profile of the FBG, $I_{R}$ is the reference arm detected intensity, $I_{S}$ is the sample arm detected intensity, $\Delta x_{S}$ is the sample displacement, $\Delta x_{R}$ is the reference effective displacement, and $k_{o}$ is the light source's center wave number. Equation (4) includes the approximation that $S(\lambda)$ is significantly wider than $B(\lambda)$ and that $B(\lambda)\left(\Delta \lambda_{\mathrm{FBG}}=\right.$ $20 \mathrm{~nm})$ is spectrally flat. A signal trace acquired in this configuration is shown in Fig. 2(a). The matched FBG configuration [Fig. 1(b)] reduces dispersion significantly; however, its coherence envelope [Fig. 2(a)] is significantly widened compared with the theoretically predicted envelope [Fig. 2(c)]. The interference signal envelope has a FWHM value of $317 \mu \mathrm{m}$ whereas the theoretical prediction gives a value of $52 \mu \mathrm{m}$ [calculated from Eq. (4) based on experimental parameters].

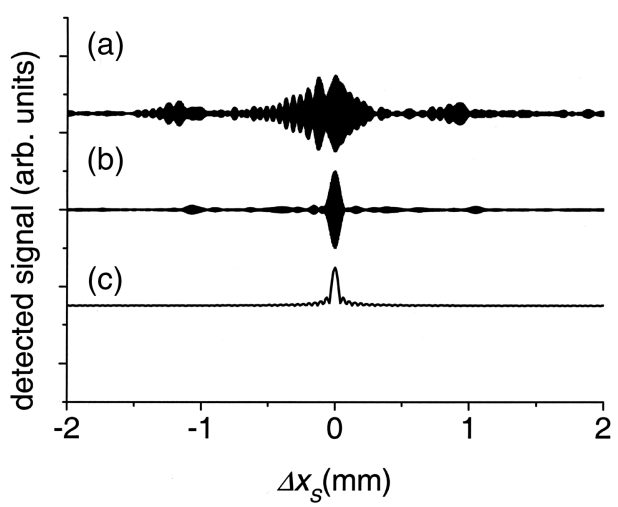

Fig. 2. Plots of detected heterodyne signals for two of the experimental configurations shown in Figs. 1(b) and 1(c) on an expanded scale: (a) detected signal for the configuration in Fig. 1(b), (b) detected signal for the configuration in Fig. 1(c), (c) theoretical signal amplitude prediction. 


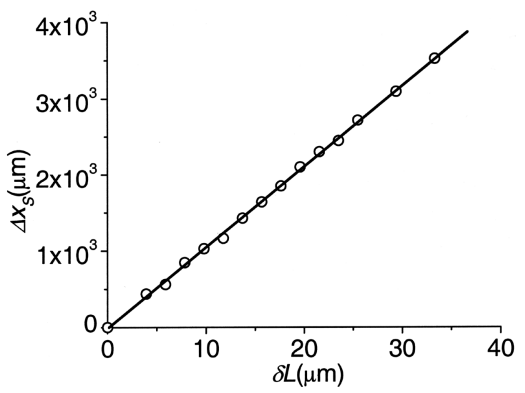

Fig. 3. Plot of the optical delay $\left(\Delta x_{S}\right)$ induced by a physical stretch $(\delta L)$ of one of the FBGs for the experimental configuration depicted in Fig. 1(b).

To investigate the potential responsibility of a slight FBG mismatch for the imperfect dispersion compensation demonstrated by use of the configuration of Fig. 1(b), we recorded signal traces using the configuration of Fig. 1(c), where a single FBG fulfills both roles of the two FBGs. The measured signal from this configuration can be found in Fig. 2(b). Since the same FBG is illuminated from both directions, the dispersion induced on the light should be close to equal and opposite, resulting in approximately zero dispersion of the final product. (Problems associated with exact dispersion cancellation can be found in Ref. 8.) In this experiment we observed a signal envelope with a FWHM value of $69 \mu \mathrm{m}$ (approximately $33 \%$ wider than the theoretical prediction). Unfortunately, this configuration cannot be used to create a variable optical delay, as stretching of the FBG in one path corresponds to compression of the FBG in the complementary path.

As previously noted in Eq. (3), the predicted path amplification is $\left(1-p_{e}\right) n_{o}\left(\lambda_{\mathrm{FBG}} / \Delta \lambda_{\mathrm{FBG}}\right)$. To verify the amplification effect, one of the FBGs of the configuration in Fig. 1(b) was progressively stretched $(\delta L)$ while the location of the interference maxima was monitored by mirror displacement $\Delta x_{S}$. A total stretch of $33 \mu \mathrm{m}$ was achieved, and the final observed optical delay shift was $3495 \mu \mathrm{m}$. The optical path delay change is plotted in Fig. 3. The observed amplification factor of 106 agrees reasonably well with the predicted amplification of 75 based on the experimental parameters mentioned.

For optical coherence tomography applications, a reflection bandwidth of more than $50 \mathrm{~nm}$ at the operat- ing wavelength of $1.3 \mu \mathrm{m}$ is desirable. From Eq. (3) we can see that this reduces the amplification factor to 32. Given that the stress breakage limit of a typical optical fiber is a fractional length change of approximately $3 \times 10^{-3}$, this implies that we need a FBG of $2.5-\mathrm{cm}$ length to attain a variable optical translation of $2.4 \mathrm{~mm}$.

In conclusion, we have demonstrated that a matched pair of linearly chirped FBGs can be used to construct an optical delay line that is capable of large optical delay change $(3.5 \mathrm{~mm})$ actuated by small physical stretch or compression $(33 \mu \mathrm{m})$. This new technique opens up the possibility for making compact and low maintenance variable optical delay lines. The quality of the processed light depends critically on a match of the two FBGs used.

This research was supported by National Institutes of Health grant EB000243. We acknowledge useful suggestions from T. Sargent of the University of Toronto. C. Yang's e-mail address is chyang@caltech.edu.

*Now affiliated with the Department of Electrical Engineering, Mail Code 136-93, California Institute of Technology, 1200 East California Boulevard, Pasadena, California 91125.

\section{References}

1. M. R. Hee, J. A. Izatt, E. A. Swanson, D. Huang, J. S. Schuman, C. P. Lin, C. A. Puliafito, and J. G. Fujimoto, Arch. Ophthalmol. (Chicago) 113, 325 (1995).

2. C. A. Puliafito, M. R. Hee, C. P. Lin, E. Reichel, J. S. Schuman, J. S. Duker, J. A. Izatt, E. A. Swanson, and J. G. Fujimoto, Ophthalmology 102, 217 (1995).

3. S. Yazdanfar, A. M. Rollins, and J. A. Izatt, Opt. Lett. 25, 1448 (2000).

4. A. Sergeev, V. Gelikonov, G. Gelikonov, F. Feldchtein, K. Pravdenko, R. Kuranov, N. Gladkova, V. Pochinko, G. Petrova, and N. Nikulin, in Conference on Lasers and Electro-Optics, Vol. 15 of OSA Technical Digest Series (Optical Society of America, Washington, D.C., 1995), p. 349.

5. F. Ouellette, Opt. Lett. 12, 847 (1987).

6. B. H. Lee, T.-J. Eom, E. Choi, G. Mudhana, and C. Lee, "Novel optical delay line for optical coherence tomography system," Opt. Rev. (to be published).

7. W. W. Morey, J. R. Dunphy, and G. Meltz, Proc. SPIE 1586, 216 (1999).

8. R. Kashyap and M. de Lacerda Rocha, Opt. Commun. 153, 19 (1998). 